\title{
Reductive Cleavage of Sulfones and Sulfonamides by a Neutral Organic Super-Electron-Donor (S.E.D.) Reagent
}

\author{
Franziska Schoenebeck, John A. Murphy, ${ }^{*}$ Sheng-ze Zhou, Yoshitaka Uenoyama, Yves Miclo, and \\ Tell Tuttle* \\ WestCHEM, Department of Pure and Applied Chemistry, University of Strathclyde, Thomas Graham Building, \\ 295 Cathedral Street, Glasgow, G1 1XL, United Kingdom.
}

Received June 18,2007; E-mail: john.murphy@strath.ac.uk; tell.tuttle@strath.ac.uk

The sulfonyl group finds extensive applications in organic and medicinal chemistry both in sulfonamides, popular as robust protecting groups for amines, ${ }^{1}$ and in sulfones. ${ }^{2}$ Frequently, sulfones are introduced into synthetic schemes to assist particular transformations; further progress along the synthetic route can later require the removal of a sulfone group, and this can be achieved by reductive desulfonylation ${ }^{2,3,6}$ or, in the special cases of $\alpha$-halo- or $\beta$-acyloxysulfones, by elimination to an alkene. ${ }^{2-5}$

For reductive removal of sulfones and for reductive cleavage of classic sulfonamides, different methods ${ }^{2,3}$ use alkali metals ( $\mathrm{Li}, \mathrm{Na}$, $\mathrm{K})$, lithium naphthalenide, $\mathrm{SmI}_{2}$ with $\mathrm{HMPA}$, or $\mathrm{LiAlH}_{4}$ in the presence of nickel compounds. All these reduction methods are mediated by highly aggressive metal-containing reducing agents. Electrochemical reduction is also used for the reductive cleavage of aryl sulfones ${ }^{7}\left(\mathrm{ArSO}_{2} \mathrm{R}\right)$ and sulfonamides. ${ }^{8}$ With typical reduction potentials of $-2.3 \mathrm{~V},{ }^{8 \mathrm{~b}}$ they pose some of the greatest challenges in functional group reduction.

In this paper, we report the first reductive cleavage of sulfones and sulfonamides with a neutral organic electron donor, operating in its ground state, a member of the family of reagents that were recently named the "super-electron-donor" reagents. ${ }^{9}$ These compounds are tetraazaalkenes that on oxidation afford radical cations and dications stabilized by aromaticity.

We recently disclosed ${ }^{10}$ the ability of the super-electron-donor reagent bisbenzimidazolylidene $\mathbf{1}^{11}$ to reduce aryl iodides and alkyl iodides in excellent yields to the corresponding radicals. Although $\mathbf{1}$ is a powerful reducing agent, ${ }^{11,12}$ it was not powerful enough to reduce sulfones or sulfonamides. Accordingly, more powerful reducing agents were sought, and bisimidazolylidene $\mathbf{3}$ was selected. ${ }^{9,11}$ Compound $\mathbf{3}$ has previously been prepared ${ }^{11}$ by electrochemical reduction of the disalt 4 ; it shows a 2-electron wave $\left[E_{1 / 2}(\mathrm{DMF})^{11 \mathrm{a}}=-1.20 \mathrm{~V}\right.$ vs $\left.\mathrm{SCE}\right]$ and thus $\mathbf{3}$ is a significantly better reducing agent than 1 . We have very recently devised an easy preparation of $\mathbf{3}$ from $\mathbf{2}$ (Scheme 1), ${ }^{9}$ which, in turn, is simply formed from imidazole and diiodopropane. Although its organic chemistry had not previously been explored, we have shown ${ }^{9}$ that 3 is the first neutral organic molecule to form aryl anions from iodoarenes by double electron-transfer.

To test the reactivity of donor $\mathbf{3}$, three monosulfones were now tested. Sulfone $\mathbf{5}$ underwent conversion to the alkene $\mathbf{8}$ in high yield (79\%). Similarly, sulfone 6 was cleanly converted (97\%) into hydrocarbon 9. However, the "unactivated" sulfone 7 afforded only starting material. This made it clear that reagent 3 would selectively cleave mildly activated sulfones. The most useful group of such sulfones are the gem-disulfones; such disulfones are widely used in synthesis. ${ }^{2}$ They are normally reductively cleaved with alkali metals ${ }^{1,2}$ to the corresponding monosulfone anions 20. Accordingly, we assayed $\mathbf{3}$ against disulfones 10-13. We were pleased to isolate excellent yields (94-98\%) of the monosulfones 14-17 after

13368 a J. AM. CHEM. SOC. 2007, 129, 13368-13369
Scheme 1. Preparation of Donor 3 and Reactions with Sulfones
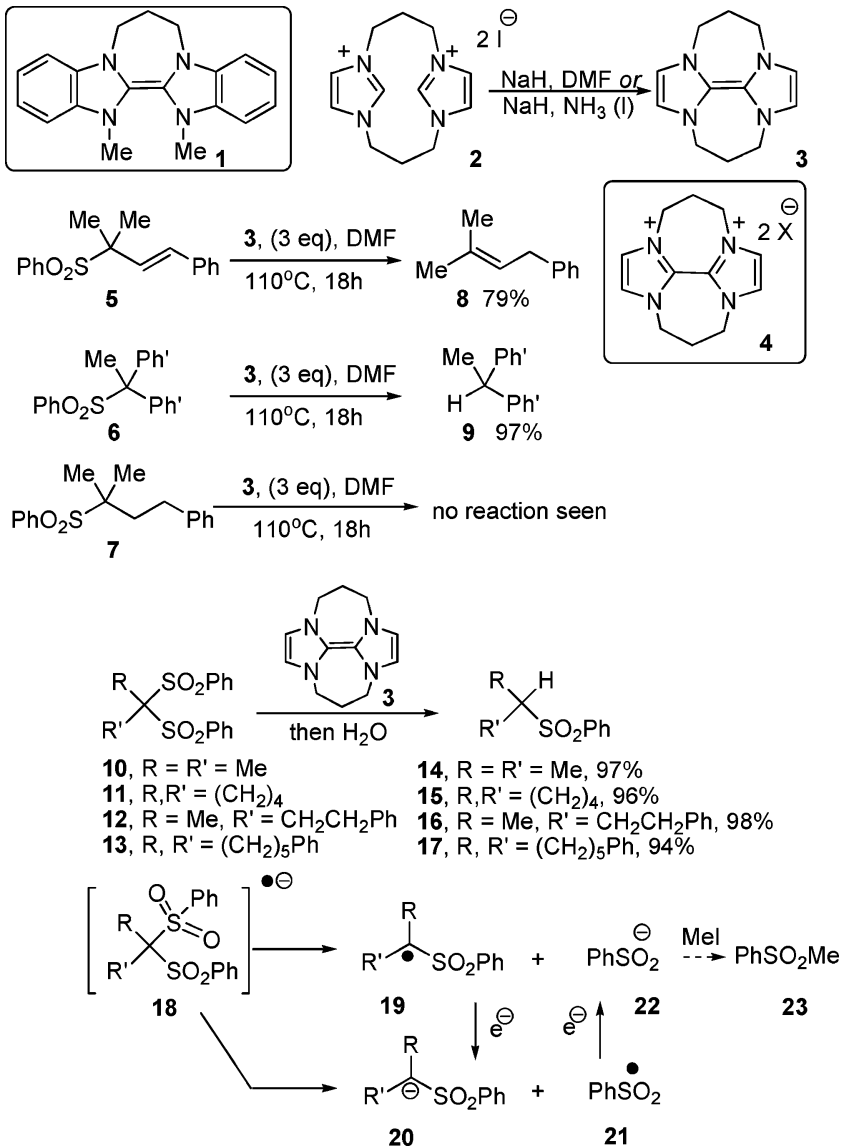

workup and chromatography. The oxidation products of $\mathbf{3}$, that is, the corresponding radical-cation (not shown) and the dication $\mathbf{4}$, are water-soluble salts, allowing easy purification of products 1417.

In these reactions, electron-transfer to the arenesulfonyl group affords the radical-anion $\mathbf{1 8}$ that could fragment to give either [radical $\mathbf{1 9}+$ anion 22] or [anion $20+$ radical 21] (see later for further discussion). Transfer of a further electron under the reaction conditions should lead to the pair of anions $\mathbf{2 0}+\mathbf{2 2}$. Confirmation of the presence of the anion $\mathbf{2 2}$ came from a repeat of the reduction experiment for substrate 11. At the end of the experiment iodomethane (excess) was added, affording phenyl methylsulfone, $\mathrm{PhSO}_{2} \mathrm{Me} 23$ (86\%).

It is known that geminal disulfones are activated relative to monosulfones, ${ }^{8 \mathrm{~b}}$ and also that aryl allylsulfones and aryl benzylsulfones $\mathrm{ArSO}_{2} \mathrm{CH}_{2} \mathrm{Ar}^{\prime}$ undergo more facile cleavage than aryl alkylsulfones $\mathrm{ArSO}_{2} \mathrm{R},{ }^{8 \mathrm{~b}}$ but analysis of the nature of the activations 
Scheme 2. Reductive Cleavage Experiments with Sulfonamides

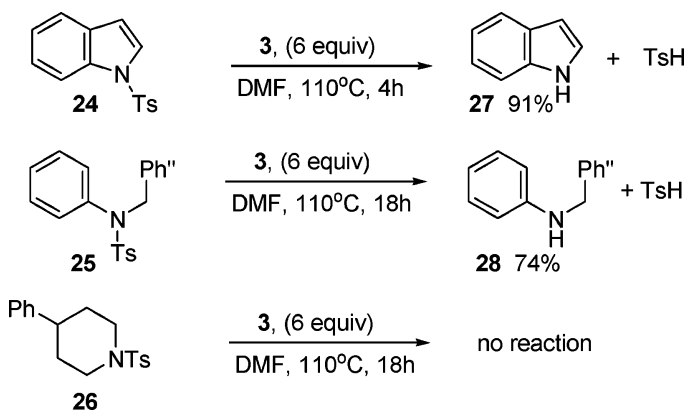

has not previously been undertaken. To understand the basis of the selectivity seen above, computational investigations were carried out that examined the nature of the electron-transfer reactions and the fragmentation of the radical anions. These studies show that the activation energy required for the electron transfer to 7 is much larger than in the case of $\mathbf{5 , 6}$, or $\mathbf{1 0}$ and that this is the crucial factor associated with nonreaction of $\mathbf{7}$ (see Supporting Information). For 5, 6, and 10, low activation energy is associated with the electron transfer and simultaneous dissociation into a sulfinate anion occurs spontaneously. This dissociation can be explained from an orbital perspective, where the LUMOs of $\mathbf{5}$ and $\mathbf{6}$ have a much greater overlap than 7 , with the $\sigma^{*}$-orbital of their respective scissile $\mathrm{C}-\mathrm{S}$ bonds (see Supporting Information). Thus, this larger overlap explains the spontaneous dissociation of the radical anions derived from $\mathbf{5}$ and $\mathbf{6}$ and the lack of cleavage for the radical anion derived from 7 .

Reductions of sulfonamides with reagent $\mathbf{3}$ were then addressed, testing three substrates 24-26 that featured different degrees of stabilization in their nitrogen leaving groups. The indolesulfonamide 24 underwent cleavage to 27 in $91 \%$ yield over $4 \mathrm{~h}$. The anilinesulfonamide $\mathbf{2 5}$ was likewise reacted and over $18 \mathrm{~h}$ gave a very satisfactory $74 \%$ yield of $\mathbf{2 8}$. However, the piperidine derivative $\mathbf{2 6}$ did not react. Computational studies show that in analogy to 7, the inactivity of $\mathbf{2 6}$ is a result of the large activation energy associated with the electron transfer, due to the instability of the radical anion (see Supporting Information for details). It has been proposed that cleavage of sulfonamide radical anions should result in aminyl radicals and sulfinate anions, ${ }^{8 b}$ but the issue has never been explored in detail. Analysis of the radical anions derived from $\mathbf{2 4}$ and 25, reveals a spontaneous reaction, akin to a fragmentation followed by a loose association of the two fragments; in each case, the associated complex might best be described as a three-electron $\mathrm{N}-\mathrm{S}$ bonded intermediate. ${ }^{13}$ For indole substrate $\mathbf{2 4}$, the charge distribution shows that the negative charge is principally associated with the indole, possibly reflecting the aromatic stabilization of the developing anion. In the case of aniline derivative 25, the intermediate shows the negative charge principally associated with the arenesulfonyl unit (see Supporting Information).

As mentioned above, typical sulfones and sulfonamides have very negative reduction potentials, typically $-2.3 \mathrm{~V}$ vs SCE. ${ }^{8 \mathrm{~b}}$ As with halides, their reduction can be achieved by electron-transfer from molecules with less negative redox potentials, provided that the radical-anion from the sulfone or sulfonamide can undergo relatively rapid fragmentation. ${ }^{8 \mathrm{~b}}$ Whereas direct electrochemical reduction of these groups requires operation at potentials close to the standard reduction potentials, indirect electrochemical reduction can be achieved $^{8 \mathrm{~b}, 14}$ by using an electrode to reduce a mediator such as pyrene $\left(E^{\circ}=-2.018 \mathrm{~V}\right)$ or anthracene $\left(E^{\circ}=-1.8908 \mathrm{~V}\right)$ to the corresponding radical-anion. However, these radical ions are charged and, as seen from their redox potentials, relatively aggressive species. In our case, reagent 3 is neutral and operates at much milder potentials $\left(E_{1 / 2}=-1.20 \mathrm{~V}\right)$. Moreover, as $\mathbf{3}$ has already been prepared from electrochemical reduction of its dication $4,{ }^{11}$ the whole process could be driven electrochemically at very mild potentials, if desired.

Reagent $\mathbf{3}$ has several advantages: (i) it is neutral; (ii) it can be conveniently prepared from imidazole and diiodopropane, followed by treatment with base; (iii) although it could be used as a reagent coupled to an electrochemical reduction, it can, as here, be completely decoupled from electrochemistry; this avoids possible complications with fouling of electrodes; (iv) $\mathbf{3}$ is used here in conventional glassware and in organic solvents without added electrolytes; (v) higher temperatures can be used than are routinely possible in electrochemical operation; (vi) since electron-donor 3 is available as a pure organic solid, the starting concentration of the reducing agent can be controlled; this contrasts with indirect electrochemistry, where the active reducing agent must be produced in situ.

In summary, donor $\mathbf{3}$ is the first neutral organic reagent to reduce arenesulfonyl groups in sulfones and sulfonamides. Unlike the alkali metals, the reagent shows pronounced selectivity for substrates that are slightly activated. The ease of preparation of these SED reagents, their mildness as neutral reagents, and the clear possibilities to modulate their structures and hence their reactivity (cf. 1 and $\mathbf{3}$ ) suggest wide applications in reductive transformations.

Acknowledgment. We thank EPSRC National Mass Spectrometry Service Centre, Swansea, for high-resolution mass spectra and EPSRC and the University of Strathclyde for funding.

Supporting Information Available: Typical experimental procedures for the preparations and reductions of substrates, spectroscopic data for the products and details of computational studies. This material is available free of charge via the Internet at http://pubs.acs.org.

\section{References}

(1) (a) Greene, T. W.; Wuts, P. G. M. Protective Groups in Organic Synthesis, 2nd ed.; J. Wiley \& Sons: New York, 1991. (b) Kocieński P. J. Protecting Groups; Georg Thieme Verlag: Stuttgart, New York, 1994.

(2) (a) Simpkins, N. S. Sulphones in Organic Synthesis; Pergamon Press: Oxford, England, 1993. (b) Magnus, P. D. Tetrahedron 1977, 33, 2019

(3) Najera, C.; Jus, M. Tetrahedron, 1999, 55, 10547.

(4) (a) Julia, M. Pure Appl. Chem., 1985, 57, 763. (b) Blakemore, P. R. J. Chem. Soc., Perkin Trans. 1 2002, 2563.

(5) (a) Taylor, R. J. K. Org. React. 2003, 62, 357. (b) Paquette, L. A. Org. React. 1977, 25, 1 .

(6) Trost, B. M.; Arndt, H. C.; Strege, P. E.; Verhoeven, T.R. Tetrahedron Lett. 1976, 17, 3477 .

(7) (a) Horner, L.; Neumann, H. Chem. Ber. 1965, 1715 (b) Delaunay, J.; Mabon, G.; Chaquiq el Badre, M.; Orliac, A.; Simonet, J. Tetrahedron Lett. 1992, 33, 2149. (c) Simonet, J. The Chemistry of Sulphones and Sulphoxides; Patai, S., Rappoport, Z., Stirling, C., Eds.; Wiley-Interscience: Chichester, England, 1988; Chapter 22.

(8) (a) Maia, H. L. S.; Medeiros, M. J.; Montenegro, M. I.; Court, D.; Pletcher, D. J. Electroanal. Chem. Interfacial Electrochem. 1984, 164, 347. (b) Lund, H. Organic Electrochemistry, 4th ed.; Lund, H., Hammerich, O. Eds.; Marcel Dekker: New York, 2001; Chapter 23. (c) Simonet, J; Pilard, J.-F. Organic Electrochemistry, 4th ed.; Lund, H., Hammerich, O., Eds.; Marcel Dekker: New York, 2001; Chapter 29.

(9) Murphy, J. A.; Zhou, S.-Z.; Thomson, D. W.; Schoenebeck, F.; Mohan, M.; Park, S. R.; Tuttle, T.; Berlouis, L. E. A. Angew. Chem., Int. Ed. 2007, 46, 5178 .

(10) Murphy, J. A.; Khan, T. A.; Zhou, S. -Z.; Thomson, D. W.; Mohan, M. Angew. Chem., Int. Ed. 2005, 44, 1356.

(11) (a) Ames, J. R.; Houghtaling, M. A.; Terrian, D. L.; Mitchell, T. A. Can J. Chem. 1997, 75, 28. (b) Thummel, R. P.; Goulle, V.; Chen, B. J. Org. Chem. 1989, 54, 3057. (c) Taton, T. A.; Chen, P. Angew. Chem., Int. Ed. Engl. 1996, 35, 1011. (d) Hünig, S.; Sheutzov, D.; Schlaf, H. Justus Liebigs Ann. Chem. 1972, 765, 126

(12) $E_{1 / 2}$ (DMF) $=-0.76,-0.82 \mathrm{~V}$ vs SCE.

(13) Brunelle, P.; Schoeneich, C.; Rauk, A. Can. J. Chem. 2006, 84, 893.

(14) Kossaï, R.; Simonet, J.; Jeminet, G. Tetrahedron Lett. 1979, 20, 1059. JA074417H 